\title{
POTENTIAL DISSEMINATION OF ANTIMICROBIAL RESISTANCE FROM SMALL SCALE POULTRY SLAUGHTERHOUSES IN PAKISTAN
}

\author{
WASEEM, H. $.^{1,3}-$ ALI, J. ${ }^{2}-$ JAMAL, A. ${ }^{1}-$ ALI, M. I. ${ }^{1 *}$ \\ ${ }^{I}$ Environmental Microbiology Laboratory, Department of Microbiology, Quaid-i-Azam \\ University, Islamabad 45320, Pakistan \\ (e-mails:waseemh1@msu.edu; asifjamall@yahoo.com)
}

${ }^{2}$ Laboratory of Environmental Nanomaterials, Research Center for Eco-Environmental Sciences, Chinese Academy of Sciences, 18 Shuangqing Road, Beijing 100085, PR China

(e-mail: Jafarali_st@rcees.ac.cn)

${ }^{3}$ Department of Biotechnology, University of Sialkot, Punjab 51310, Pakistan

*Corresponding author

e-mail: ishimrl@qau.edu.pk; phone: +92-519-064-3196

(Received $3^{\text {rd }}$ Dec 2018; accepted $4^{\text {th }}$ Feb 2019)

\begin{abstract}
The importance of environmental reservoirs as a source from which antimicrobial resistance emerges and subsequently transferred is already established. To evaluate the role of small scale poultry slaughterhouses in resistance dissemination in Pakistan, a comparative genomic analysis of antimicrobial resistance genes (ARGs) and mobile genetic elements (MGEs) in the gut microbiome of broiler and household chickens (Gallus gallus domesticus) was performed. An array of 52 qPCR primers targeting the 16S rRNA gene, ARGs and MGEs was used in the experiment to analyse the abundance of selected genes in collected samples. A total of 22 ARGs and 7 MGEs were detected in all samples. The detection frequency of specific gene classes and diversity of genes was found to be higher in broiler as compared to household chicken. Sull was the most abundant ARG with the highest percent relative abundance (2.4\%). Total percent relative abundance of ARGs in broiler chicken was found to be significantly $(\mathrm{p}<0.05)$ greater than household chicken fecal samples. A significant linear correlation $\left(\mathrm{R}^{2}=0.89\right)$ was found between relative abundances of int 1 and total ARGs. The clustering and correlation of selective ARGs with MGEs has implied that small scale poultry slaughterhouses can be a potential source for the dissemination of ARGs to other non-resistant environmental and/or clinical bacteria.
\end{abstract}

Keywords: $A R G s, M G E s, q P C R, A M R$, microbiome

\section{Introduction}

Antimicrobial resistance genes (ARGs) are emerging environmental contaminants causing serious public health concerns (Sanderson et al., 2016). The annual number of human deaths due to antimicrobial resistance is expected to reach up to 10 million by 2050 (de Kraker et al., 2016). Accumulating scientific evidence have suggested that the abuse of antibiotics in clinical and agricultural settings is mainly responsible for the emergence of antimicrobial resistance in microorganisms (Rather et al., 2017; York, 2017). Antibiotic resistant bacteria and associated ARGs can be transported from different environmental reservoirs contributing to the ever increasing global problem of antimicrobial resistance (Waseem et al., 2017a). The scientific community is already exploring new directions for evaluating the spread of the antimicrobial resistance from environmental routes to clinical pathogens (Andersson, 2015; Waseem et al., 2019; Williams et al., 2017). Fecal microflora from animals and birds harbours a vast variety 
of ARGs that can be transferred into other bacteria including human pathogens (Bonnedahl and Järhult, 2014; Zhao et al., 2018).

The poultry industry is one of the fastest growing agricultural sectors of the world. Use of antibiotics at therapeutic and sub-therapeutic levels as growth promoters have been employed in poultry for over half a century (Castanon, 2007). Although antibiotics utility for growth promotion has already been ostracized in many developed countries, but the situation is disquieting in developing countries like Pakistan and Egypt (Mund et al., 2017). In Pakistan, over the counter availability of antibiotics and less implementation of health and safety regulations in the poultry industry makes the challenge of antibiotic abuse and subsequent resistance dissemination far more intriguing. By 2030, the global consumption of antibiotics for cattle, chicken, and pigs is expected to reach 105,596 $\pm 3,605$ tons (Van Boeckel et al., 2015). The consumption of antibiotics is continuously putting increasing pressure on environmental and clinical bacteria to acquire antimicrobial resistance. Apart from the resistance development under selection pressure for antibiotics, bacteria can also develop resistance by acquiring resistance genes via mobile genetic elements (MGEs) mediated horizontal gene flow (Oliveira et al., 2017).

One of the most useful strategies against the global antimicrobial resistance is to mitigate the spread of ARGs from environmental reservoirs. Surveillance and monitoring of such reservoirs are thus essential for curbing the dissemination of ARGs into the environment. Small scale poultry slaughterhouses are one of the potential reservoirs for antibiotic resistance. They are widely distributed throughout Pakistan. Chicken fecal waste from these small scale slaughterhouses, a source of resistant bacteria and ARGs, is generally being disposed of in the environment without any proper treatment. Chances of meat contamination with the antimicrobial resistant bacteria at the time of slaughtering in unhygienic conditions are also there (Amir et al., 2017). Additionally, ARGs can also be disseminated into the environment when poultry feces are used as organic fertilizers for fruits and vegetables.

Antibiotics can also influence ARGs to which they are not explicitly related (Foxman, 2012; Guo et al., 2018). Therefore, instead of antibiotic specific screening of ARGs in chicken (Gallus gallus domesticus) gut microbiota, analysis of ARGs from all major classes is recommended. Additionally, most of the research on antimicrobial resistance was focused on individual bacteria mainly foodborne pathogens (Lee et al., 2017; McMahon et al., 2007), which represents only a small fraction of the gut microbiome. Evaluation of resistance determinants from gut microbiome community can give a comprehensive analysis of dissemination and/ or fate of the ARGs into the environment.

To the best of our knowledge the status of AMR in gut microbiome of chicken in Pakistan yet remains elucidative to a greater extent. The primary purpose of the current study was to evaluate and compare the detection frequency, diversity, and abundance of selected ARGs from chicken fecal samples collected from small scale poultry slaughterhouses. The abundance and occurrence of selected MGEs have also been evaluated as they are believed to be involved in the dispersal of ARGs into non-resistant pathogenic bacterial strains. The possible correlations between ARGs and MGEs were also determined. Present research is a pilot scale study with only a limited sample set but it will likely provide first-hand information regarding ARGs dissemination from the small scale poultry slaughterhouses. 


\section{Materials and methods}

\section{Sample collection and DNA extraction}

Sample collection was performed in early spring of 2018 from a small scale poultry slaughter house in Johar Town, Lahore, Pakistan. Pools of fresh fecal samples from different cages of broiler and household chickens were collected (Fig. Al in the Appendix). A total of 6 fecal samples 3 from broiler chickens and 3 from household chickens were collected in $30 \mathrm{ml}$ falcon tubes using a sterile spatula. Samples were thoroughly mixed and homogenized before DNA extraction (Fig. 1). DNA was extracted from collected samples by using QIAGEN DNeasy PowerSoil kit (QIAGEN Inc., MD, USA) as per manufacturer's instruction. Extracted DNA was stored at $-20{ }^{\circ} \mathrm{C}$ until the amplification was performed. All the experiments in our study were performed at Environmental Microbiology Laboratories, Department of Microbiology, Quaid-iAzam University, Islamabad. The concentration of DNA from each sample was normalized to $10 \mathrm{ng} / \mu \mathrm{l}$ before using the DNA for qPCR reactions.

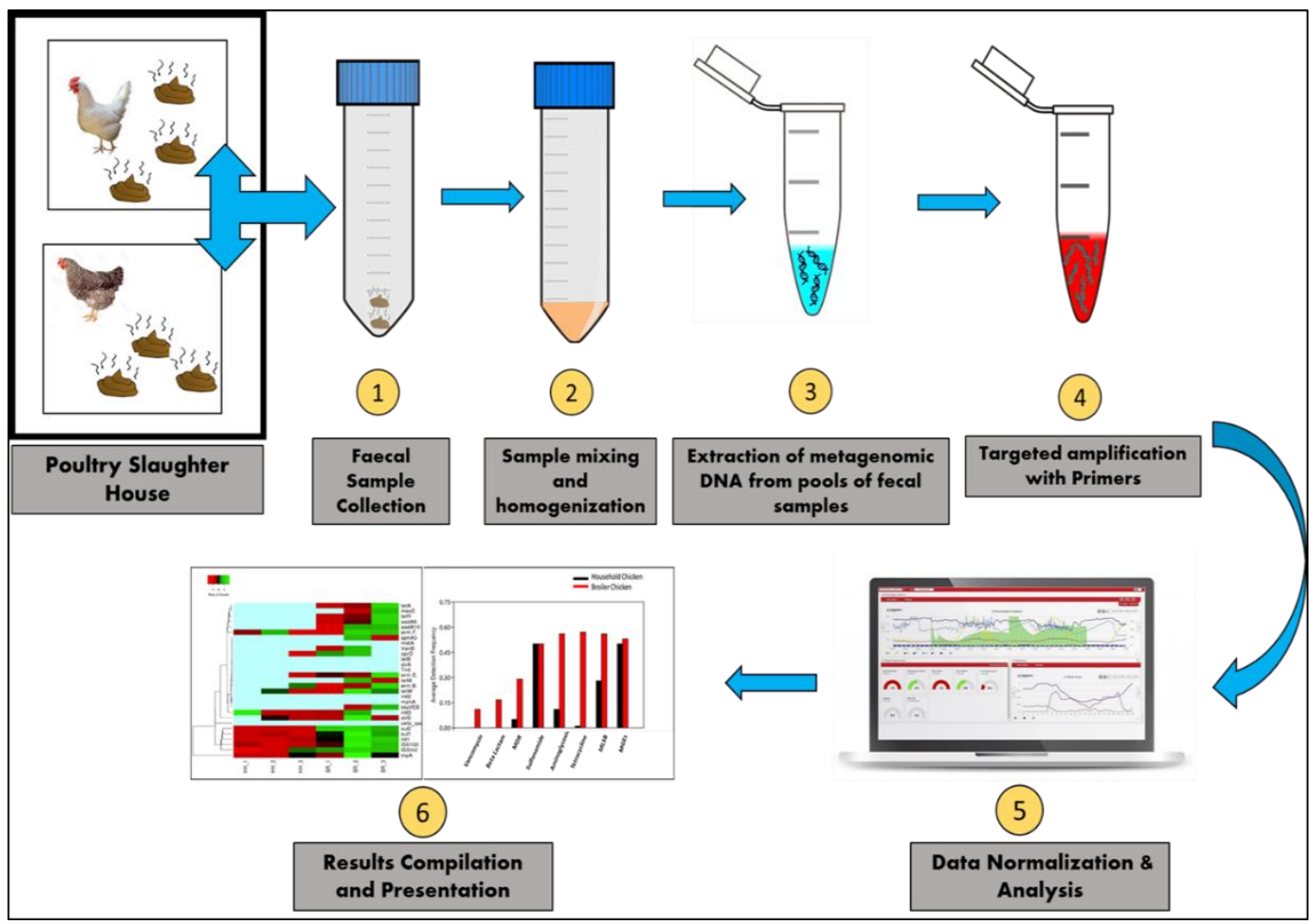

Figure 1. Diagrammatic flowchart of the whole experimental design

\section{Primers and $q P C R$}

A total of 52 primers were used in this study for the evaluation of ARGs and MGEs abundance in fecal samples. The primers used in our study are a subset of qPCR Primer set 2.0 (Stedtfeld et al., 2018). The sequences of the qPCR primers can be found in the Appendix (Table A1). The primers were designed, validated and assayed as described in previous studies (Johnson et al., 2016; Karkman et al., 2016; Stedtfeld et al., 2017, 2018). The design protocol and parameters of each primer set are to target sequence diversity within a gene to analyse the environmental resistome. The array covered seven 
major antibiotic classes including MDR ( 7 genes), tetracyclines (7 genes), aminoglycosides (6 genes) beta-lactams (6 genes), macrolide-lincosamidestreptogramin B (MLSB) (6 genes), Vancomycin (6 genes) sulfonamides (3 genes) involved in different resistance mechanisms (Fig. 2).

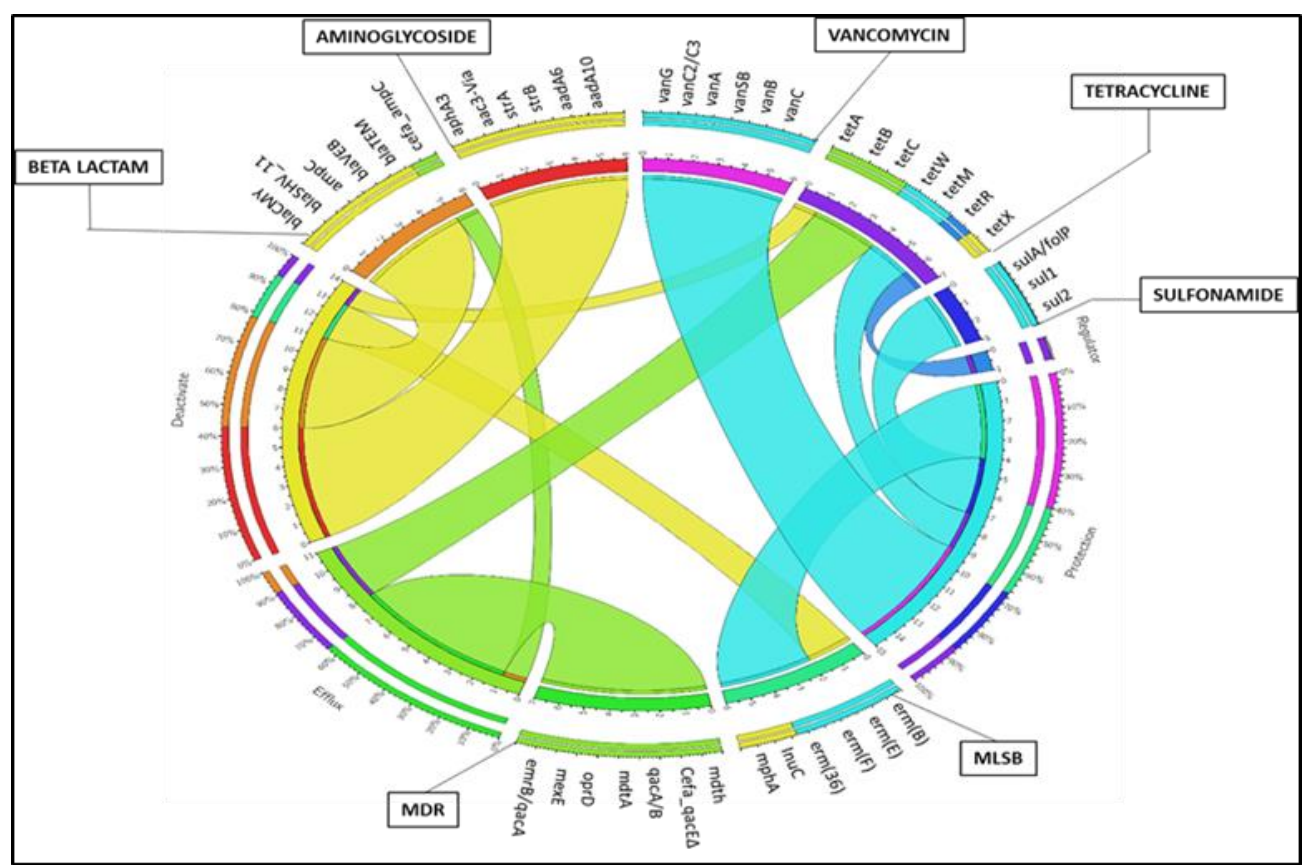

Figure 2. Circos plot showing the antibiotic resistance class and mode of resistance mechanism of all 41 ARGs included in our array. The plot is made by using Cir-cos software (Krzywinski et al., 2009)

A real-time qPCR thermocycler (Mastercycler ${ }^{\circledR}$ ep realplex; Eppendorf) was used to perform DNA amplification in $25 \mu \mathrm{l}$ reaction volume. The contents used in qPCR are as follows: 2x Power SYBR Green PCR Master Mix (12.5 $\mu \mathrm{l}), 10 \mu \mathrm{m}$ primer mix $(1.25 \mu \mathrm{l})$, sample DNA $(1 \mu \mathrm{l})$, and water $(10.25 \mu \mathrm{l})$. Cycling conditions for qPCR reactions were as follow: an initial activation step at $95{ }^{\circ} \mathrm{C}$ for $10 \mathrm{~min}$ followed by 45 cycles of $95^{\circ} \mathrm{C}$ for $15 \mathrm{~s}$ and $60^{\circ} \mathrm{C}$ for $1 \mathrm{~min}$. Gene copy number was estimated from the Cycle threshold $(\mathrm{Ct})$ values based on the method previously described (Looft et al., 2012). A Ct value of 29 was used as the cut-off limit. All sample-primer assays were performed in triplicates. A negative control without sample DNA was also included for every assay. Genes detected from at least two out of three technical replicates were considered as true positive and all false positive results were removed. Overall a total of 1248 qPCR reactions were performed during the whole study.

\section{Data analysis}

Relative abundance was calculated by dividing the estimated gene copy number of the targeted gene with the gene copy number of $16 \mathrm{~S}$ rRNA. A multivariate principal component analysis (PCA) of ARG profiles between sample types was performed in Paleontological Statistics Software Package for Education and Data Analysis (PAST: v3.20) (Hammer et al., 2001). The scale of the principal components was transformed into eigenvalues. Basic data processing of qPCR results was performed on Microsoft 
Excel 2010. A student t-test was applied to the sum of total ARGs and MGEs abundance in order to test the significant differences between two different types of chicken. Shannon diversity calculations and scatter plots were also made in PAST v3.20 using percent relative abundance of genes in order to see the diversity of the ARGs in different samples. A heat map based on the natural log-transformed values of the relative abundance of ARGs and MGEs was made by using heat mapper software (Babicki et al., 2016) to evaluate the presence of individual genes in fecal samples. Complete linkage clustering method and Euclidean distance measurement method was used to cluster the rows representing genes in the heat map (Yeung et al., 2003).

\section{Results}

\section{Detection and diversity of ARGs and MGEs}

For evaluating the ARGs of 6 fecal samples from 2 different types of chicken (broiler vs. household), a qPCR sub-array of 52 primer sets targeting 16S rRNA gene, antibiotic resistance genes (ARGs) and transposase genes related to horizontal gene transfer (HGT) were used in the present study (Fig. 2). Out of 52 targeted genes, a total of 22 ARGs were detected in the fecal samples. The detected ARGs represented every targeted class of antibiotics (Fig. 3). In addition to ARGs 7 MGEs were also detected in both groups. The detected MGEs belonged to insertion sequences, transposase, and integrase groups are believed to play a role in HGT. Out of 29 detected ARGs and MGEs 8 genes were detected all (6/6) samples while 6 genes were detected in only 1 out of 6 samples. Two genes conferring resistance to aminoglycosides (aadA6, aadA10) and two tetracycline resistant genes $($ tet $A$, tet $R$ ) were absent in all three samples from household chicken but were detected in all three samples from broiler chicken. Overall more genes were detected in broiler chicken samples as compared to household chicken samples.

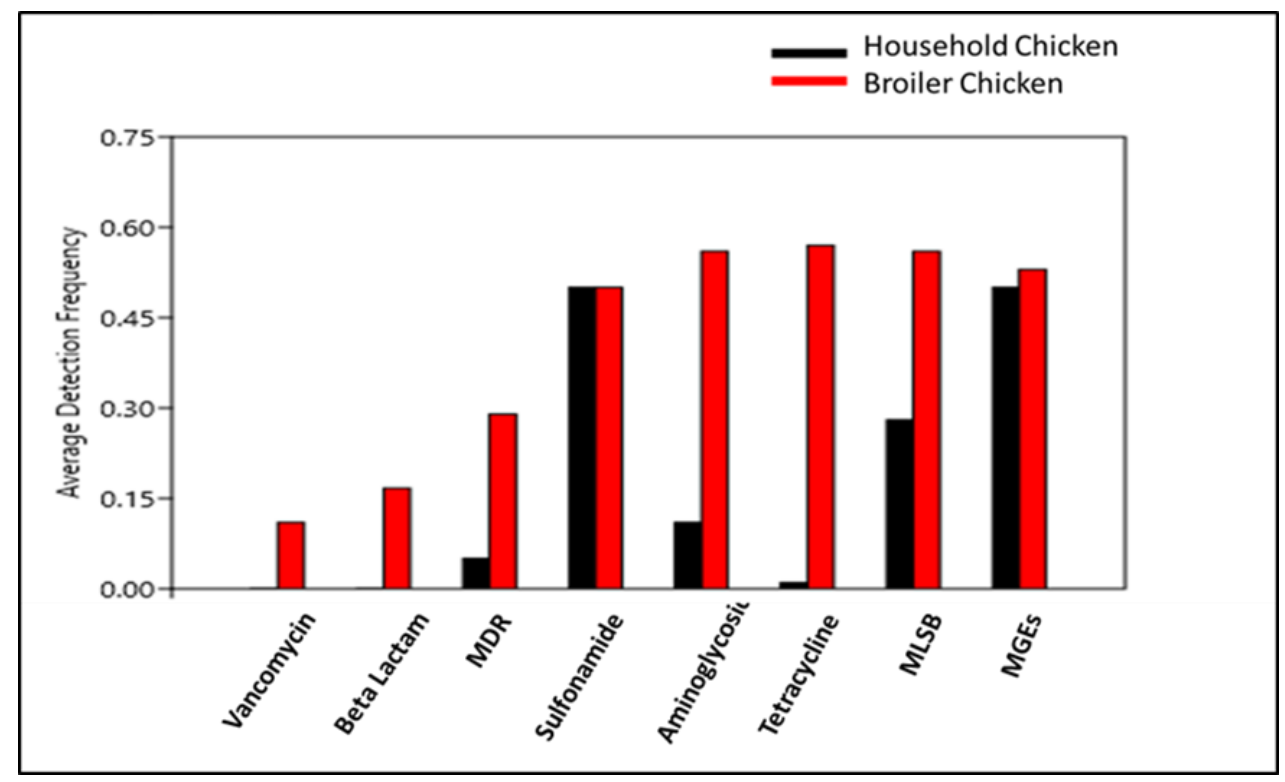

Figure 3. Antibiotic resistance class wise average detection frequency. Average detection frequency is an average number of samples in which genes within a class is detected divided by total number of samples against which the genes are tested for one or more genes conferring resistance to that AR class 
The number of detected genes ranged from 8 to 13 in fecal samples from household chicken whereas the genes detected in fecal samples of broiler chicken ranged from 18 to 24 . Shannon diversity indicating the richness and abundance of genes in the samples was calculated by taking an average of the relative abundance of the three technical replicates for each of the 6 samples. Shannon diversity of the broiler chicken samples was found to be higher than the household chicken (Fig. 4).

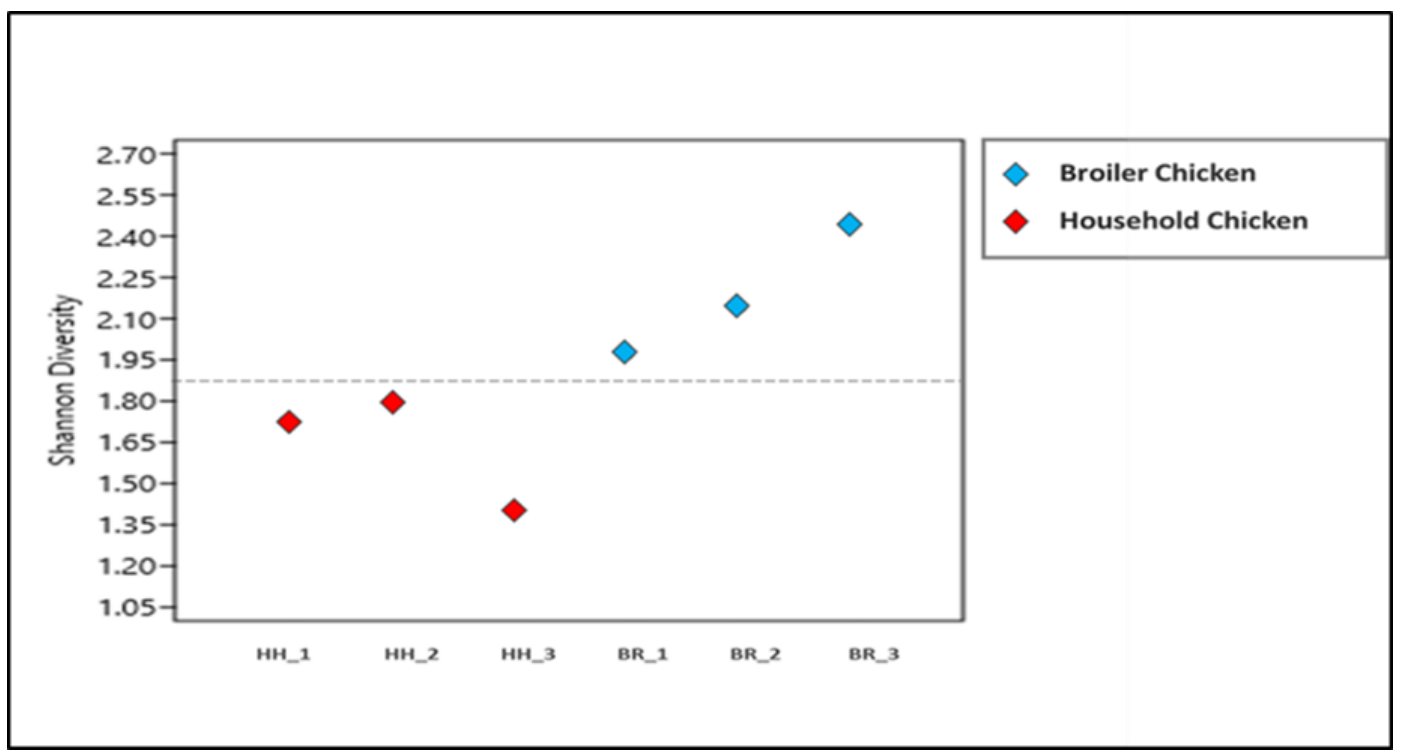

Figure 4. Scatter plot showing the Shannon diversity of the ARGs and MGEs in 6 fecal samples

\section{The abundance of the ARGs and MGEs}

To minimize the sampling variations, the estimated gene copy number of ARGs and MGEs was normalized to $16 \mathrm{~S}$ rRNA gene copy number to achieve the relative abundance. The relative abundance of different genes varied significantly among the samples (Fig. 5a). The most abundant gene was tnpA, a transposase from IS-6 family detected in all 6 samples with percent relative abundance ranging from 0.52 to 4.68 whereas the least abundant gene was an ARG vanB from vancomycin group, the only gene detected from this group, ranged from $2.4 \times 10^{-3}$ to $1.3 \times 10^{-2}$. Overall the summed relative abundance of ARGs $(\mathrm{p}<0.05)$ and MGEs was higher in broiler chicken fecal samples than in broiler chicken samples (Fig. 5b).

Gene cluster patterns were observed by using ordination analysis. Relative abundances of the ARGs were used to perform multivariate PCA analysis in order to assess the distribution of ARGs among two chicken types (broiler vs. household). The PCA plot has revealed that the ARGs profile of fecal samples from two chicken groups is different from each other (Fig. 6).

Correlations among different gene classes were also evaluated in our study. Significant correlations were observed between total abundances of aminoglycosides, sulphonamides and tetracycline with int 1 and/ or total MGEs. The highest coefficient of determination $\left(\mathrm{R}^{2}\right)$ values was observed for aminoglycosides with MGEs and int1 (Table 1). The abundance of intl was also considerably correlated to the total relative abundance of the ARGs based on the linear regression analysis (Fig. 7). 


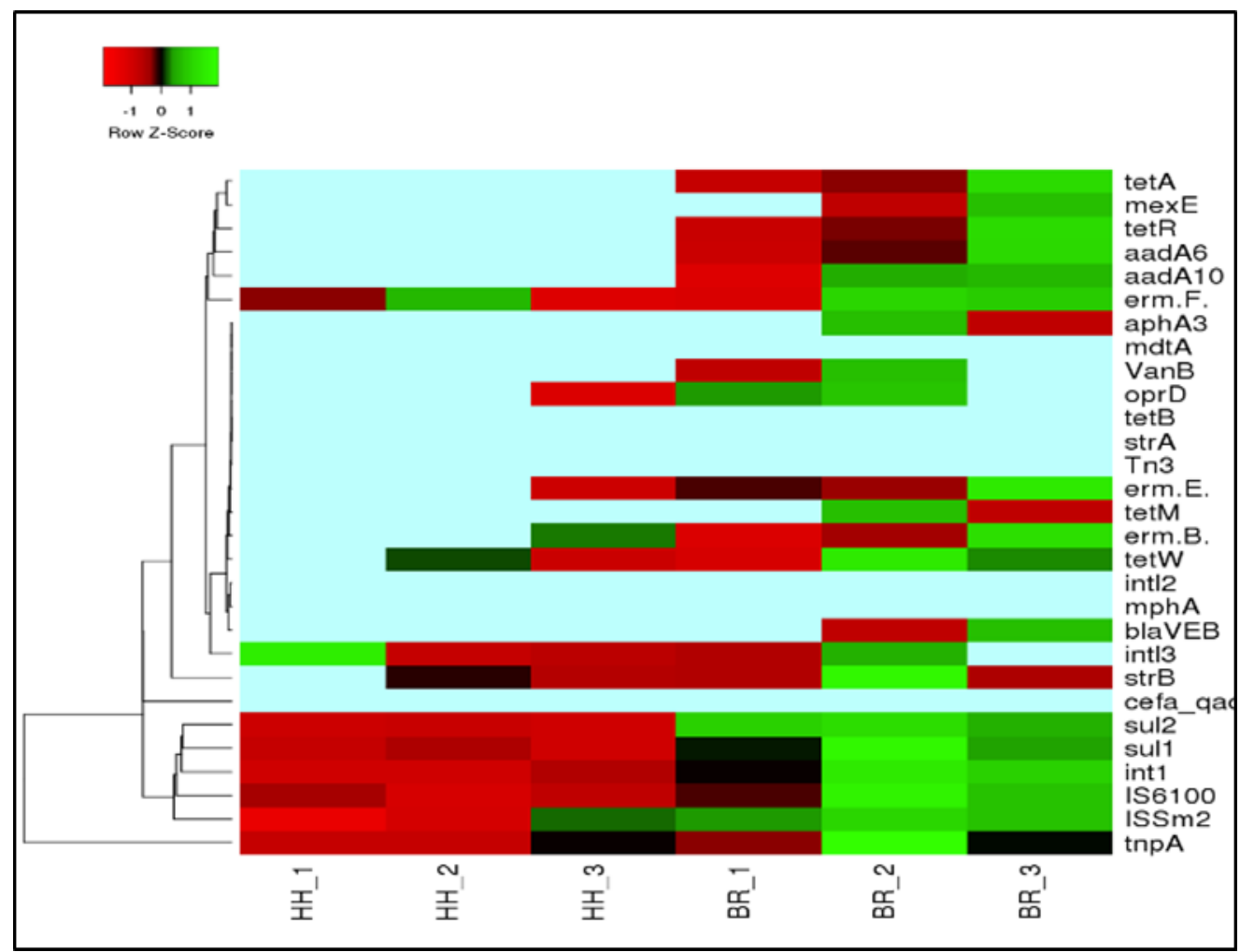

A

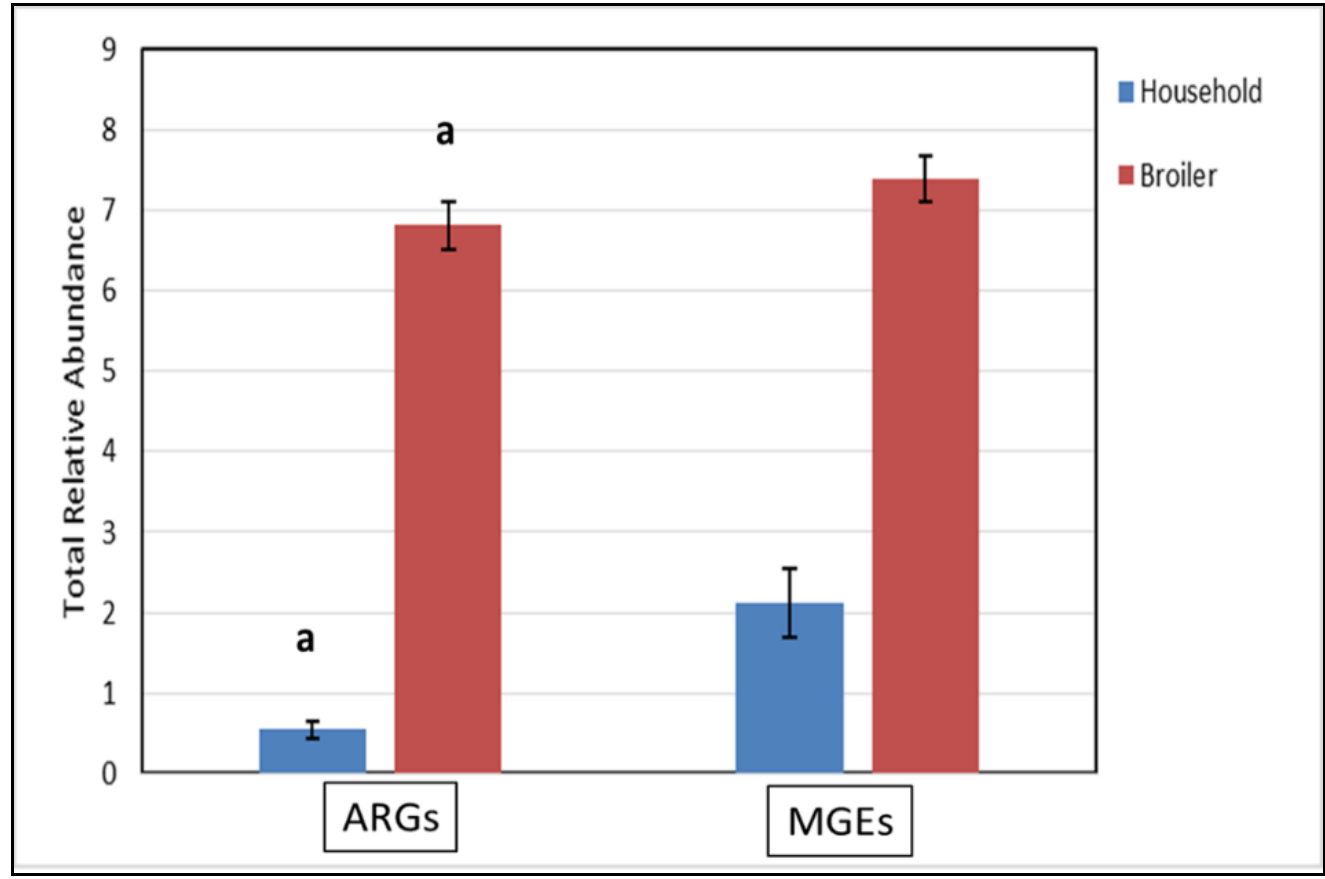

B

Figure 5. a Heat Map showing the ARGs and MGEs detected in 6 fecal samples ( 3 technical replicates each). Relative abundance (log transformed) was used for the formation of the heat map. Complete linkage clustering is showing that the sul genes are clustered with many MGEs including int1. $\boldsymbol{b}$ Sum of percent relative abundances of ARGs and MGEs in two types of chicken measured by 6 pools of fecal samples (3 technical replicates each). Letter (a) shows that the difference is significant at 95\% confidence of interval. Error bars are showing the standard error of the calculated mean 


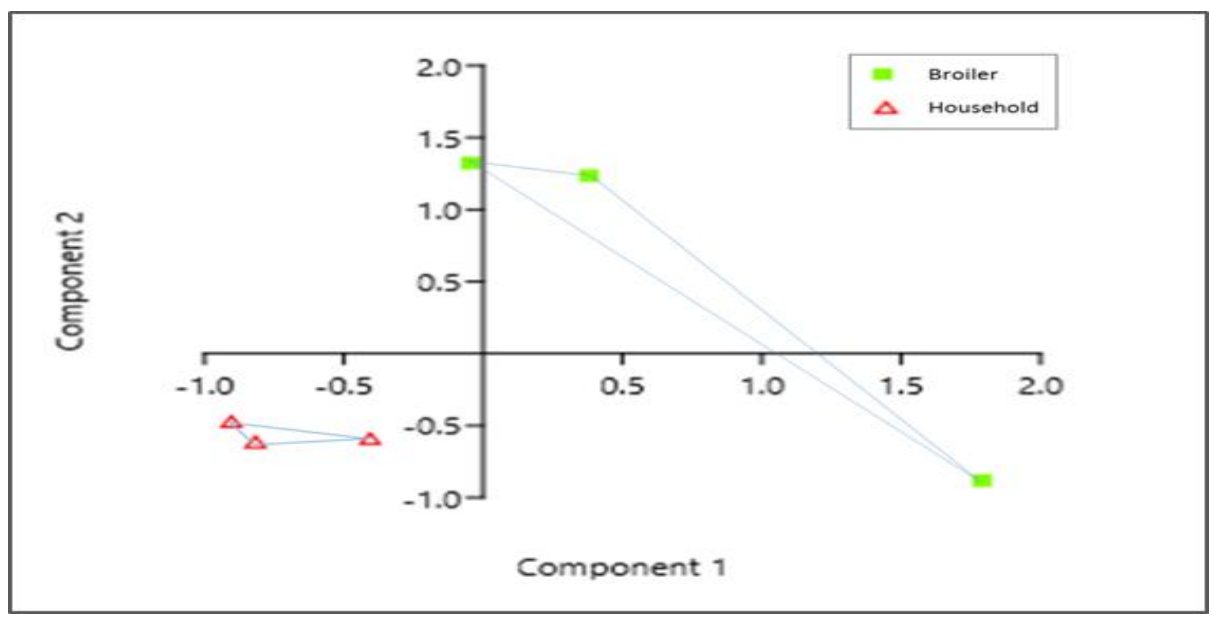

Figure 6. Ordination of ARGs with 6 fecal samples from two different chicken types. Red triangles represented fecal samples of household chickens. Green squares represented fecal samples from broiler chickens

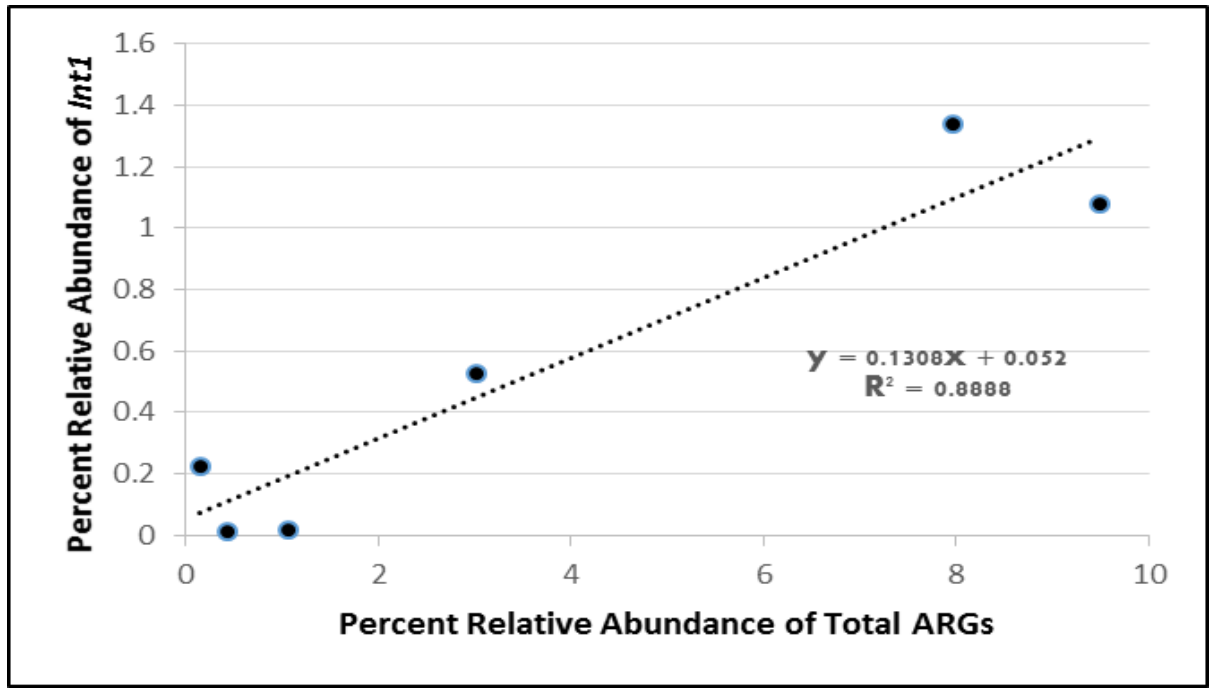

Figure 7. Linear regression curve is showing a significant correlation between intl and total ARGs abundances in 6 samples

Table 1. Coefficient of determination $R^{2}$ values between different gene classes

\begin{tabular}{c|c|c|c}
\hline Sr. no. & Gene class 1 & Gene class 2 & $\mathbf{R}^{2}$ value \\
\hline 1 & Total ARGs & Total MGEs & 0.67 \\
2 & Aminoglycoside & int1 & 0.93 \\
3 & Aminoglycoside & MGEs & 0.88 \\
4 & Sulfonamide & Int1 & 0.85 \\
5 & Sulfonamide & MGEs & 0.78 \\
6 & Tetracycline & int1 & 0.53 \\
7 & Tetracycline & MGEs & 0.29 \\
8 & MDR & int1 & 0.26 \\
9 & MDR & MGEs & 0.08 \\
10 & MLSB & Int1 & 0.4 \\
11 & MLSB & MGEs & 0.25 \\
\hline
\end{tabular}




\section{Discussion}

A large body of literature has provided information regarding the threats of antimicrobial resistance using conventional methods (Álvarez-Fernández et al., 2013; Khalili et al., 2012). In recent years, dissemination of ARGs via environmental routes has gained much attention. Characterization of mobile genetic elements along with other genes is becoming essential in antimicrobial resistance research (Waseem et al., 2017b). In our study, we have opted a relatively broader and comprehensive approach of targeting multiple genes (ARGs and MGEs) in the gut metagenomes of broiler and household chickens (Gallus gallus domesticus). Our data is useful for assessing the role of small scale poultry slaughterhouses, which are widely distributed throughout the country, in ARGs dissemination. Most of the conventional small scale slaughterhouses have improper blood and wastewater drainage system (Sohaib and Jamil, 2017) which further intensifies the threat of ARGs dissemination into the environment.

Fecal samples from broiler chickens, which get routine antimicrobial treatments, were found to have higher abundance and diversity of ARGs compared to the household chickens which had been on organic antibiotic-free feed. Our results are in accordance with many different studies where the resistance abundance or detection frequency of resistant genes was significantly higher in antibiotics treated chickens, pigs and cattle as compared to the controls (Cameron and McAllister, 2016; Guo et al., 2018; Looft et al., 2012). Our findings demonstrated that the detection frequency of MGEs was almost equal in two sample types but the total percent relative abundance was higher in broiler chicken. Similar results of elevated MGEs were also observed in small scale production birds as compared to household birds during a study of small scale production poultry operations in rural Ecuador (Moser et al., 2018). This signifies that apart from the selection of ARGs under the selection pressure of antibiotics, higher abundance of MGEs could also be a critical factor in the transfer of genes within chromosomes, across bacteria and even in between different species (Stokes and Gillings, 2011).

The most frequently detected ARGs (at least 5/6 samples) in our study were sull, $s u l 2$, ermF, strB, tet $W$. The presence of ARGs in household chicken type is not surprising. The same genes were also detected in Gallus gallus domesticus feces and many other environmental matrices without antibiotic treatment (Berglund, 2015; Sui et al., 2016; Zhang et al., 2013). This implies some resistance genes could be ubiquitously present in the environment without antibiotic's selection pressure. For example, a diverse intrinsic antibiotic resistome was recently studied in bacteria isolated from a cave (Pawlowski et al., 2016). Such genes can provide a competitive advantage to environmental bacteria harbouring them over others.

Sulfonamide resistance in bacteria usually arises by acquiring sul genes, encoding dihydropteroate synthase that is not inhibited by the antibiotics (Antunes et al., 2005). Sul genes have been most consistent targets in poultry and animal live stocks studies (Kozak et al., 2009; McKinney et al., 2010). Both of these genes were detected in all 6 samples. Considerable differences were found in the relative abundance of these genes within each sample. The difference in the abundance of these closely related genes can be ascribed to the differences in their dissemination mechanism. Sul2 is normally located on Inc. Q family of plasmids whereas sull is believed to be distributed with the help of integrons (He et al., 2014). Similar observation of different relative abundance of these related genes has been reported in other environmental matrices as well (Cheng et al., 2013; Sui et al., 2016; Zhang et al., 2013). A study had reported the prevalence and distribution of sulfonamide resistance genes in soils around poultry and livestock 
farms in Jiangsu Province, Southeastern China. Distribution frequency of sul genes and most frequent combination of sul1 and sul2 was studied (Wang et al., 2014). Our results are in close agreement with this study as the type of animal and waste can affect the distribution of ARGs in the environment.

Transposon specific gene tnpA, integron specific gene intl along with two other MGEs have been clustered together with sulphonamide resistant genes (Fig. 4a). Transposons and integrons have been investigated in-silico (Loot et al., 2017) and also in environmental matrices (Stokes and Gillings, 2011) to evaluate the possible enrichment of ARGs. TnpA association with antibiotic resistance is also highlighted in many studies (Carnelli et al., 2017; Feng et al., 2017). The int 1 gene is believed to be responsible for the evolution and dissemination of multiple antibiotic resistant bacteria and the spread of mobile genetic elements in the environments (Moura et al., 2012). In our recent publication int 1 gene as a proxy for environmental surveillance of ARGs has also been reconfirmed (Stedtfeld et al., 2017). An important observation in our present study is the strong correlation among the relative abundance of int 1 gene and total ARGs. The reason behind this correlation could be co-occurrence of int 1 and ARGs on a single plasmid and/ or chromosome. Our results are supported by the finding of a recent study about distribution and characterization of class 1 integrons where it was reported that these integrons harboured genes belonging to class aminoglycosides (Sung and $\mathrm{Oh}, 2014)$.

tnpA and int 1 genes were two of the most abundant genes in broiler chicken which is in accordance with the results from a study of ARGs analysis in production chicken in rural Ecuador where the relative abundance of these two genes were highest among others (Guo et al., 2018). The clustering of intI1 and tnpA with ARGs has implied that broiler chickens in small scale slaughterhouses can negatively play their role by spreading ARGs in clinically important strains and thus can produce multiple antibiotic resistant strains which can cause serious public health concerns. For example, Talebiyan and colleagues have isolated 318 multiple resistant E. coli from commercial broiler flocks that have been given different doses of antibiotics (Talebiyan et al., 2014). This signifies that the broiler chickens in small scale slaughterhouses can provide an appropriate environment for the proliferation of ARGs through horizontal and/ or vertical gene transfer. Our results about the abundance and detection of ARGs in chicken fecal samples are in accordance with the trends of ARGs abundance observed in other gut microbiomes (Waseem et al., 2019).

Concrete measures should be opted in order to mitigate the threat of antimicrobial resistance. In this regard, strict AMR centric environmental strategies should be implemented on commercial poultry farms so that the use of antibiotics in the poultry industry should be regulated. Small scale poultry slaughterhouses, especially located in clusters, should be encouraged to develop their own waste and water management systems. Use of untreated chicken feces and litter as fertilizer should be discouraged. Apart from formulating and implementing rules and regulations, general public awareness campaigns about antimicrobial stewardship should be launched. This can also increase the demand for organic meat and/ or household chicken thus forcing the poultry farm owners to restrict antimicrobials use as growth promoters. 


\section{Conclusion}

Our study has provided an effective assessment and potential insight into the role and potential risk of small scale poultry slaughterhouses with the perspective of antimicrobial resistance. More clinically important ARGs were detected from the samples collected from cages of broiler chicken. Broiler chicken mostly raised in large scale commercial farms are usually located in outskirts of communities. Their transport poses a risk in the introduction of exogenous ARGs in the environment since such small scale poultry slaughterhouses are distributed widely throughout the country. Our research will aid in curbing the dissemination of antimicrobial resistance into the environment from such environmental reservoirs. Further research with larger sample size, more ARG and MGE targets along with bacterial community analysis will be helpful for validating the trends of ARGs spread in the environment.

Acknowledgements. The authors are very grateful to Dr. Maggie Williams from Michigan State University, East Lansing, Michigan, USA and Dr. Robert Stedtfeld from Swift Biosciences, Ann Arbor, Michigan, USA for their continuous support and assistance.

\section{REFERENCES}

[1] Álvarez-Fernández, E., Cancelo, A., Díaz-Vega, C., Capita, R., Alonso-Calleja, C. (2013): Antimicrobial resistance in E. coli isolates from conventionally and organically reared poultry: A comparison of agar disc diffusion and Sensi Test Gram-negative methods. - Food Control 30(1): 227-234.

[2] Amir, M., Riaz, M., Chang, Y. F., Akhtar, S., Yoo, S. H., Sheikh, A. S., Kashif, M. (2017): Impact of unhygienic conditions during slaughtering and processing on spread of antibiotic resistant Escherichia coli from poultry. - Microbiological Research (Pavia) 8(2): 7330.

[3] Andersson, D. I. (2015): Improving predictions of the risk of resistance development against new and old antibiotics. - Clinical Microbiology and Infection 21(10): 894-898.

[4] Antunes, P., Machado, J., Sousa, J. C., Peixe, L. (2005): Dissemination of sulfonamide resistance genes (sul1, sul2, and sul3) in Portuguese Salmonella enterica strains and relation with integrons. - Antimicrobial Agents and Chemotherapy 49(2): 836-9.

[5] Babicki, S., Arndt, D., Marcu, A., Liang, Y., Grant, J. R., Maciejewski, A., Wishart, D. S. (2016): Heatmapper: web-enabled heat mapping for all. - Nucleic Acids Research 44(W1): W147-W153.

[6] Berglund, B. (2015): Environmental dissemination of antibiotic resistance genes and correlation to anthropogenic contamination with antibiotics. - Infection Ecology Epidemiology 5: 28564.

[7] Boeckel, T. P. Van., Brower, C., Gilbert, M., Grenfell, B. T., Levin, S. A., Robinson, T. P., Teillant, A., Laxminaraya, R. (2015): Global trends in antimicrobial use in food animals. Proceedings of the National Academy of Sciences of USA 112(18): 5649-5654.

[8] Bonnedahl, J., Järhult, J. D. (2014): Antibiotic resistance in wild birds. - Upsala Journal of Medical Sciencies 119(2): 113-6.

[9] Cameron, A., McAllister, T. A. (2016): Antimicrobial usage and resistance in beef production. - Journal of Animal Science and Biotechnology 7(1): 68.

[10] Carnelli, A., Mauri, F., Demarta, A. (2017): Characterization of genetic determinants involved in antibiotic resistance in Aeromonas spp. and fecal coliforms isolated from different aquatic environments. - Research in Microbiology 168(5): 461-471.

[11] Castanon, J. I. R. (2007): History of the use of antibiotic as growth promoters in European poultry feeds. - Poultry Science 86(11): 2466-2471. 
[12] Cheng, W., Chen, H., Su, C., Yan, S. (2013): Abundance and persistence of antibiotic resistance genes in livestock farms: A comprehensive investigation in eastern China. Environmental International 61: 1-7.

[13] Danish Mund, M., Hassan Khan, U., Tahir, U., Fayyaz, A. (2017): Antimicrobial drug residues in poultry products and implications on public health: A review. - International Journal of Food Properties 20(7): 1433-1446.

[14] Feng, J., Yin, Z., Zhao, Q., Zhao, Y., Zhang, D., Jiang, X., Wu, W., Chen, W, Wang, H., Song, Y., Tong, Y., Wang, J., Li, Y., Zhou, D. (2017): Genomic characterization of novel IncFII-type multidrug resistant plasmids p0716-KPC and p12181-KPC from Klebsiella pneumoniae. - Scientific Reports 7(1): 5830.

[15] Foxman, B. (2012): Applications of Molecular Tools to Infectious Disease Epidemiology. 1st Ed. - Academic Press, Cambridge, MA.

[16] Guo, X., Stedtfeld, R. D., Hedman, H., Eisenberg, J. N. S., Trueba, G., Yin, D., Tiedje, J. M., Zhang, L. (2018): Antibiotic resistome associated with small-scale poultry production in rural Ecuador. - Environmental Science \& Technology 52(15): 8165-8172.

[17] Hammer, Ø., Harper, D. A. T., Ryan, P. D. (2001): PAST: paleontological statistics software package for education and data analysis. - Palaeontologia Electronica 4(1): 1-9.

[18] He, L. Y., Liu, Y. S., Su, H. C., Zhao, J. L., Liu, S. S., Chen, J., Liu, W. R., Ying, G. G. (2014): dissemination of antibiotic resistance genes in representative broiler feedlots environments: Identification of indicator ARGs and correlations with environmental variables. - Environmental Science \& Technology 48(22): 13120-13129.

[19] Johnson, T. A., Stedtfeld, R. D., Wang, Q., Cole, J. R., Hashsham, S. A., Looft, T., Zhu, Y. G., Tiedje, J. M. (2016): Clusters of antibiotic resistance genes enriched together stay together in swine agriculture. - MBio 7(2): e02214-15.

[20] Karkman, A., Johnson, T. A., Lyra, C., Stedtfeld, R. D., Tamminen, M., Tiedje, J. M., Virta, M. (2016): High-throughput quantification of antibiotic resistance genes from an urban wastewater treatment plant. - FEMS Microbiology Ecology 92(3): fiw014.

[21] Khalili, H., Soltani, R., Negahban, S., Abdollahi, A., Gholami, K. (2012): Reliability of disk diffusion test results for the antimicrobial susceptibility testing of nosocomial Grampositive microorganisms: Is E-test method better? - Iranian Journal of Pharmaceutical Research 11(2): 559-63.

[22] Kozak, G. K., Pearl, D. L., Parkman, J., Reid-Smith, R. J., Deckert, A., Boerlin, P. (2009): Distribution of sulfonamide resistance genes in Escherichia coli and Salmonella isolates from swine and chickens at abattoirs in Ontario and Québec, Canada. - Applied and Environmental Microbiology 75(18): 5999-6001.

[23] Kraker, M. E. A. de., Stewardson, A. J., Harbarth, S. (2016): Will 10 million people die a year due to antimicrobial resistance by 2050? - PLoS Medicine 13(11): e1002184.

[24] Krzywinski, M., Schein, J., Birol, I., Connors, J., Gascoyne, R., Horsman, D., Jones, S. J., Marra, M. A. (2009): Circos: an information aesthetic for comparative genomics. Genome Research 19(9): 1639-45.

[25] Lee, J. H., Shin, D., Lee, B., Lee, H., Lee, I., Jeong, D. W. (2017): Genetic diversity and antibiotic resistance of Enterococcus faecalis isolates from traditional Korean fermented soybean foods. - Journal of Microbiology and Biotechnology 27(5): 916-924.

[26] Looft, T., Johnson, T. A., Allen, H. K., Bayles, D. O., Alt, D. P., Stedtfeld, R. D., Sul, W. J., Stedtfeld, T. M., Chai, B., Cole, J. R., Hashsham, S. A., Tiedje, J. M., Stanton, T. B. (2012): In-feed antibiotic effects on the swine intestinal microbiome. - Proceedings of the National Academy of Sciences of USA 109(5): 1691-6.

[27] Loot, C., Nivina, A., Cury, J., Escudero, J. A., Ducos-Galand, M., Bikard, D., Rocha, E. P. C., Mazel, D. (2017): Differences in integron cassette excision dynamics shape a tradeoff between evolvability and genetic capacitance. - MBio 8(2): e02296-16.

[28] McKinney, C. W., Loftin, K. A., Meyer, M. T., Davis, J. G., Pruden, A. (2010): tet and sul antibiotic resistance genes in livestock lagoons of various operation type, 
configuration, and antibiotic occurrence. - Environmental Science and Technology 44(16): 6102-6109.

[29] McMahon, M. A. S., Xu, J., Moore, J. E., Blair, I. S., McDowell, D. A. (2007): Environmental stress and antibiotic resistance in food-related pathogens. - Applied and Environmental Microbiology 73(1): 211-7.

[30] Moser, K. A., Zhang, L., Spicknall, I., Braykov, N. P., Levy, K., Marrs, C. F., Foxman, B., Trueba, G., Cevallos, W., Goldstick, J., Trostle, J., Eisenberg, J. N. S. (2018): The role of mobile genetic elements in the spread of antimicrobial-resistant Escherichia coli from chickens to humans in small-scale production poultry operations in rural Ecuador. American Journal of Epidemiology 187(3): 558-567.

[31] Moura, A., Pereira, C., Henriques, I., Correia, A. (2012): Novel gene cassettes and integrons in antibiotic-resistant bacteria isolated from urban wastewaters. - Research in Microbiology 163(2): 92-100.

[32] Oliveira, P. H., Touchon, M., Cury, J., Rocha, E. P. C. (2017): The chromosomal organization of horizontal gene transfer in bacteria. - Nature Communications 8(1): 841.

[33] Pawlowski, A. C., Wang, W., Koteva, K., Barton, H. A., McArthur, A. G., Wright, G. D. (2016): A diverse intrinsic antibiotic resistome from a cave bacterium. - Nature Communications 7: 13803.

[34] Rather, I. A., Kim, B. C., Bajpai, V. K., Park, Y. H. (2017): Self-medication and antibiotic resistance: Crisis, current challenges, and prevention. - Saudi Journal of Biological Sciences 24(4): 808-812.

[35] Sanderson, H., Fricker, C., Brown, R. S., Majury, A., Liss, S. N. (2016): Antibiotic resistance genes as an emerging environmental contaminant. - Environmental Reviews 24(2): 205-218.

[36] Sohaib, M., Jamil, F. (2017): An insight of meat industry in Pakistan with special reference to halal meat: a comprehensive review. - Korean Journal of food Sciences and Animal Resources 37(3): 329-341.

[37] Stedtfeld, R. D., Stedtfeld, T. M., Waseem, H., Fitschen-Brown, M., Guo, X., Chai, B., Williams, M. R., Shook, T., Logan, A., Graham, A., Chae, J. C., Sul, W. J., VanHouten, J., Cole, J. R., Zylstra, G. J., Tiedje, J. M., Upham, B. L., Hashsham, S. A. (2017): Isothermal assay targeting class 1 integrase gene for environmental surveillance of antibiotic resistance markers. - Journal of Environmental Management 198(1): 213-220.

[38] Stedtfeld, R. D., Guo, X., Stedtfeld, T. M., Sheng, H., Williams, M. R., Hauschild, K., Gunturu, S., Tift, L., Wang, F., Howe, A., Chai, B., Yin, D., Cole, J. R., Tiedje, J. M., Hashsham, S. A. (2018): Primer set 2.0 for highly parallel qPCR array targeting antibiotic resistance genes and mobile genetic elements. - FEMS Microbiology Ecology 94(9): fiy 130.

[39] Stokes, H. W., Gillings, M. R. (2011): Gene flow, mobile genetic elements and the recruitment of antibiotic resistance genes into Gram-negative pathogens. - FEMS Microbiology Reviews 35(5): 790-819.

[40] Sui, Q., Zhang, J., Chen, M., Tong, J., Wang, R., Wei, Y. (2016): Distribution of antibiotic resistance genes (ARGs) in anaerobic digestion and land application of swine wastewater. - Environmental Pollution 213: 751-759.

[41] Sung, J. Y., Oh, J. E. (2014): Distribution and characterization of integrons in Enterobacteriaceae isolates from chickens in Korea. - Journal of Microbiology and Biotechnology 24(7): 1008-13.

[42] Talebiyan, R., Kheradmand, M., Khamesipour, F., Rabiee-Faradonbeh, M. (2014): Multiple antimicrobial resistance of Escherichia coli isolated from chickens in Iran. Veterinary Medicine International 2014: 491418.

[43] Wang, N., Yang, X., Jiao, S., Zhang, J., Ye, B., Gao, S. (2014): Sulfonamide-resistant bacteria and their resistance genes in soils fertilized with manures from Jiangsu Province, Southeastern China. - PLoS One 9(11): e112626.

[44] Waseem, H., Williams, M. R., Stedtfeld, R. D., Hashsham, S. A. (2017a): Antimicrobial resistance in the environment. - Water Environment Research. 89(10): 921-941. 
[45] Waseem, H., Williams, M. R., Stedtfeld, T., Chai, B., Stedtfeld, R. D., Cole, J. R., Tiedje, J. M., Hashsham, S. A. (2017b): Virulence factor activity relationships (VFARs): a bioinformatics perspective. - Environmental Sciences Processes \& Impacts 19(3): 247-260.

[46] Waseem, H., Jameel, S., Ali, J., Saleem Ur Rehman, H., Tauseef, I., Farooq, U., Jamal, A., Ali, M. I. (2019): Contributions and challenges of high throughput qPCR for determining antimicrobial resistance in the environment: A critical review. - Molecules 24(1): 163.

[47] Williams, M. R., Stedtfeld, R. D., Waseem, H., Stedtfeld, T., Upham, B., Khalife, W., Etchebarne, B., Hughes, M., Tiedje, J. M., Hashsham, S. A. (2017): Implications of direct amplification for measuring antimicrobial resistance using point-of-care devices. Analytical Methods 9(8): 1229-1241.

[48] York, A. (2017): Bacterial evolution: Historical influences on antibiotic resistance. Nature Reviews Microbiology 15(10): 576-577.

[49] Yeung, K. Y., Medvedovic, M., Bumgarner, R. E. (2003): Clustering gene-expression data with repeated measurements. - Genome Biology 4(5): R34.

[50] Zhang, Y., Zhang, C., Parker, D. B., Snow, D. D., Zhou, Z., Li, X. (2013): Occurrence of antimicrobials and antimicrobial resistance genes in beef cattle storage ponds and swine treatment lagoons. - Science of the Total Environment 463-464: 631-638.

[51] Zhao, Y., Su, J. Q., An, X. L., Huang, F. Y., Rensing, C., Brandt, K. K., Zhu, Y. G. (2018): Feed additives shift gut microbiota and enrich antibiotic resistance in swine gut. Science of the Total Environment 621: 1224-1232.

\section{APPENDIX}

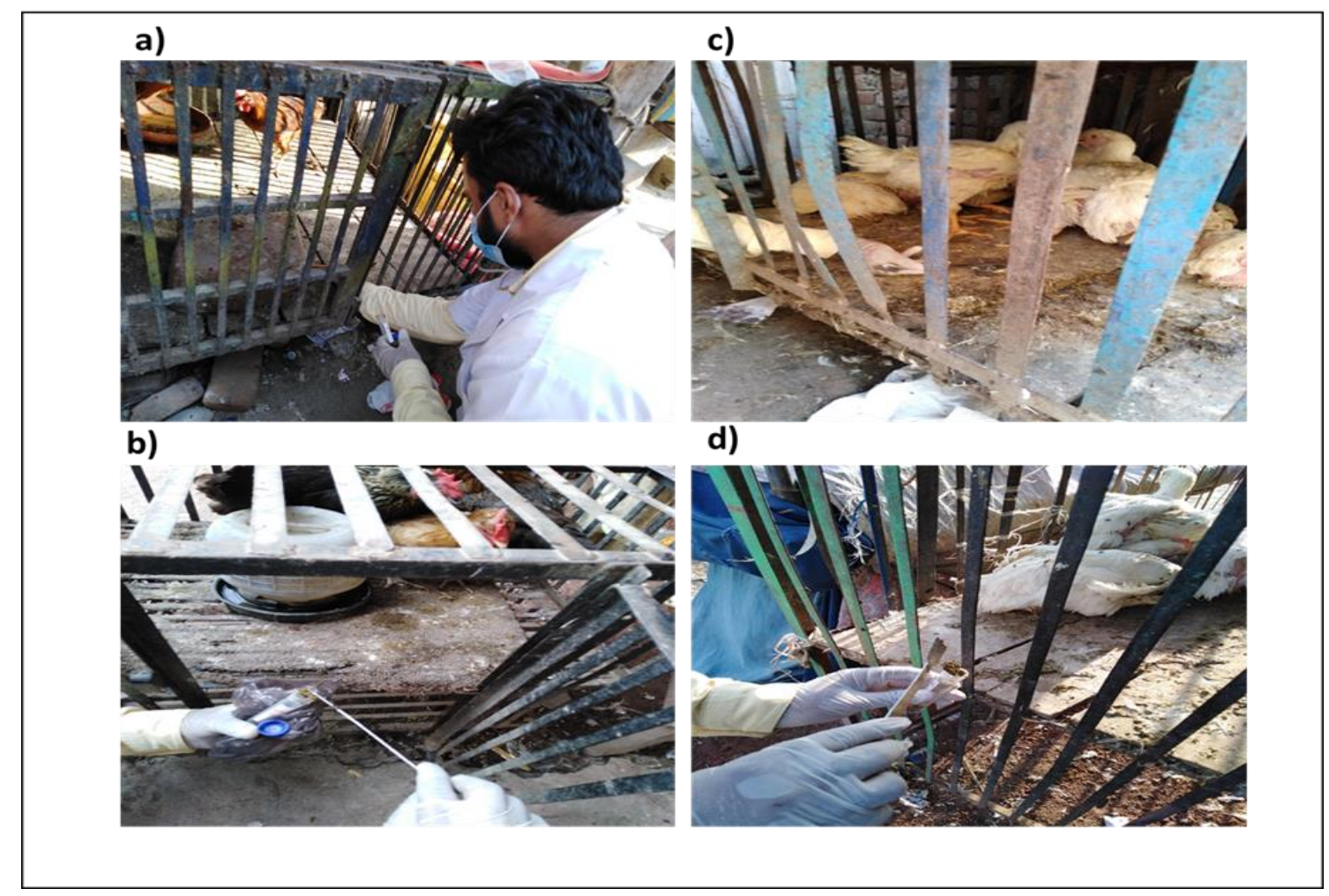

Figure A1. $\boldsymbol{a} \& \boldsymbol{b}$ Collection and transfer of household chicken fecal samples in centrifuge tubes; $\boldsymbol{c} \& \boldsymbol{d}$ Collection and transfer of broiler chicken fecal samples in centrifuge tubes 
Table A1. Primer sequences of $16 \mathrm{~S}$ rRNA gene, 41 ARGs and 10 MGEs

\begin{tabular}{|c|c|c|c|}
\hline Sr. no. & Targeted gene & Forward sequence & Reverse sequence \\
\hline 1 & 16S rRNA & GGGTTGCGCTCGTTGC & ATGGYTGTCGTCAGCTCGTG \\
\hline 2 & vanG & TGTTTCGCAGAACCGTGTCAA & CCCTGCACTGTTCCATCTTCTC \\
\hline 3 & vanC2/vanC3 & TGACTGTCGGTGCTTGTGA & GATAGAGCAGCTGAGCTTGTTC \\
\hline 4 & $\operatorname{van} \mathrm{A}$ & GGGCTGTGAGGTCGGTTG & TTCAGTACAATGCGGCCGTTA \\
\hline 5 & $\operatorname{vanSB}$ & GAAGATAAAGAGGGAAGCGTACTC & CCGAATTGTCAGCCCTTGATAA \\
\hline 6 & $\operatorname{VanB}$ & TTGTCGGCGAAGTGGATCA & AGCCTTTTTCCGGCTCGTT \\
\hline 7 & $\operatorname{vanC}$ & CCTGCCACAATCGATCGTT & CGGCTTCATTCGGCTTGATA \\
\hline 8 & blaCMY & AAAGCCTCATGGGTGCATAAA & ATAGCTTTTGTTTGCCAGCATCA \\
\hline 9 & cefa_ampc & CAGGATCTGATGTGGGAGAACTA & TCGGGAACCATTTGTTGGC \\
\hline 10 & blaSHV-11 & TTGACCGCTGGGAAACGG & TCCGGTCTTATCGGCGATAAAC \\
\hline 11 & ampC & CTGGCGCATACCTGGATTAC & GCCAGTTCAGCATCTCCCA \\
\hline 12 & blaVEB & CCCGATGCAAAGCGTTATG & GAAAGATTCCCTTTATCTATCTCAGACAA \\
\hline 13 & blaTEM & CGCCGCATACACTATTCTCAG & GCTTCATTCAGCTCCGGTTC \\
\hline 14 & mdth & ATGCTGGCTGTACAAGTGATG & CACTCCAGCGGGCGATA \\
\hline 15 & cefa_qacEdelta & TAGTTGGCGAAGTAATCGCAAC & TGCGATGCCATAACCGATTATG \\
\hline 16 & $\mathrm{qacA} / \mathrm{B}$ & AAGGGCCACTGCATTAGCTG & CCAGTCCAATCATGCCTGCA \\
\hline 17 & mdtA & ACAAGCCCAGGGCCAAC & CCTTAATGGTGCCTTCGGTTTC \\
\hline 18 & oprD & ATGAAGTGGAGCGCCATTG & GGCCACGGCGAACTGA \\
\hline 19 & $\operatorname{mexE}$ & GGTCAGCACCGACAAGGTCTAC & AGCTCGACGTACTTGAGGAACAC \\
\hline 20 & sulA/folP & CAGGCTCGTAAATTGATAGCAGAAG & CTTTCCTTGCGAATCGCTTT \\
\hline 21 & sul2 & TCATCTGCCAAACTCGTCGTTA & GTCAAAGAACGCCGCAATGT \\
\hline 22 & sul1 NEW & GCCGATGAGATCAGACGTATTG & CGCATAGCGCTGGGTTTC \\
\hline 23 & aphA3 & AAAAGCCCGAAGAGGAACTTG & CATCTTTCACAAAGATGTTGCTGTCT \\
\hline 24 & aac3-Via & GTGTCCGTCGCCAAGGA & GGTGACGGCCTTGTCGA \\
\hline 25 & strA & CCGGTGGCATTTGAGAAAAA & GTGGCTCAACCTGCGAAAAG \\
\hline 26 & $\operatorname{strB}$ & GCTCGGTCGTGAGAACAATCT & CAATTTCGGTCGCCTGGTAGT \\
\hline 27 & aadA6 & CCATCGAGCGTCATCTGGAA & CCCGTCTGGCCGGATAAC \\
\hline 28 & $\operatorname{aadA10}$ & ACAGGCACTCAACGTCATCG & CGCGGAGAACTCTGCTTTGA \\
\hline 29 & tetA & CTCACCAGCCTGACCTCGAT & CACGTTGTTATAGAAGCCGCATAG \\
\hline 30 & tetB & AGTGCGCTTTGGATGCTGTA & AGCCCCAGTAGCTCCTGTGA \\
\hline 31 & tetW & ATGAACATTCCCACCGTTATCTTT & ATATCGGCGGAGAGCTTATCC \\
\hline 32 & tet $\mathrm{X}$ & AAATTTGTTACCGACACGGAAGTT & CATAGCTGAAAAAATCCAGGACAGTT \\
\hline 33 & tetC & ACTGGTAAGGTAAACGCCATTGTC & ATGCATAAACCAGCCATTGAGTAAG \\
\hline 34 & tetR & CCGTCAATGCGCTGATGAC & GCCAATCCATCGACAATCACC \\
\hline 35 & tetM & GGAGCGATTACAGAATTAGGAAGC & TCCATATGTCCTGGCGTGTC \\
\hline 36 & $\operatorname{lnuC}$ & GGGTGTAGATGCTCTTCTTGGA & CTTTACCCGAAAGAGTTTCTACCG \\
\hline 37 & $\mathrm{emrB} / \mathrm{qacA}$ & СТTTTCTCTAACCGTACATTATCTACGATAAA & AGAACGTAGCGACTGATAAAATGCT \\
\hline 38 & $\operatorname{erm}(\mathrm{B})$ & GAACACTAGGGTTGTTCTTGCA & CTGGAACATCTGTGGTATGGC \\
\hline 39 & $\operatorname{erm}(E)$ & GTCACGCAGCTGGAGTTCG & CGGTGAAGCACAGCTCGAC \\
\hline 40 & $\mathrm{mphA}$ & TCAGCGGGATGATCGACTG & GAGGGCGTAGAGGGCGTA \\
\hline 41 & $\operatorname{erm}(F)$ & TCTGATGCCCGAAATGTTCAAG & TGAAGGACAATTGAACCTCCCA \\
\hline 42 & $\operatorname{erm}(36)$ & GGCGGACCGACTTGCAT & TCTGCGTTGACGACGGTTAC \\
\hline 43 & int13 & CAGGTGCTGGGCATGGA & CCTGGGCAGCATCACCA \\
\hline 44 & ISCR1 & ATGGTTTCATGCGGGTT & CTGAGGGTGTGAGCGAG \\
\hline 45 & $\operatorname{Tn} 3$ & GCTGAGGTGTTCAGCTACATCC & GCTGAGGTAGTCACAGGCATTC \\
\hline 46 & IS6/257 & ATATCGTGCCATTGATGCAGAG & ACCATTGCTACCTTCGTTGAAG \\
\hline 47 & $\operatorname{tnpA}$ & CCGATCACGGAAAGCTCAAG & GGCTCGCATGACTTCGAATC \\
\hline 48 & int 1 & CGAAGTCGAGGCATTTCTGTC & GCCTTCCAGAAAACCGAGGA \\
\hline 49 & intl2 & TGCTTTTCCCACCCTTACC & GACGGCTACCCTCTGTTATCTC \\
\hline 50 & ISPps1 & CACACTGCAAAAACGCATCCT & TGTCTTTGGCGTCACAGTTCTC \\
\hline 51 & ISSm2 & TGGATCGACCGGTTCCAT & GCTGACCGAGCTGTCCATGT \\
\hline 52 & IS1111 & GTCTTAAGGTGGGCTGCGTG & CCCCGAATCTCATTGATCAGC \\
\hline
\end{tabular}

